Trauma Berufskrankh 2007 - 9[Suppl 3]:

S333-S335

DOI 10.1007/s10039-007-1226-3

Online publiziert: 26. Mai 2007

(c) Springer Medizin Verlag 2007

F. Kleinschmidt

Möhnesee

\title{
Chirurgische Organisationsformen und berufsgenossenschaftliches Regelwerk
}

\author{
Was passt zusammen?
}

\begin{abstract}
Als niedergelassener Chirurg - und zwar als einziger im heutigen Programm - auf dieser BG-Tagung referieren zu dürfen, ist eine große, aber auch schwierige Ehre. Es zeigt aber auch, dass für Herrn Prof. Muhr der niedergelassene D-Arzt in das Behandlungskonzept der Verunfallten mit einzubeziehen ist.
\end{abstract}

Beim ersten Überlegen zum Thema „Chirurgische Organisationsformen und berufsgenossenschaftliches Regelwerk - was passt zusammen?" denkt man: „Alles“, jedoch sind im Lauf der Jahre Verwerfungen aufgetreten, die früher nicht vorhanden waren:

- Die Unfallversicherungsträger halten zwar gern an den Anforderungen nach $\$_{34}$ SGB VII fest, insbesondere, wenn damit Einsparungen möglich sind, nehmen aber die geforderten, strukturellen und prozessualen Anforderungen nicht unbedingt wahr.

- Die Patienten begrüßen alles, was in den Anforderungen steht, so lange es ihnen medizinisch und sozial hilft, vorausgesetzt, es bereitet nicht allzu viel persönliche Mühe.

- Die Chirurgen akzeptieren vieles, abhängig vom Ort bzw. der Praxis, dem Krankenhaus oder der Klinik sowie dem Leistungserbringer.

Im vorliegenden Beitrag soll aus der Sicht eines seit über 25 Jahren niedergelassenen D-Arztes aufzuzeigen versucht werden, dass das berufsgenossenschaftliche Regelwerk in einigen Punkten nicht unbedingt mit modernen, chirurgischen Organisationsformen kompatibel ist.

\section{Diskrepanz zwischen Regelwerk und Realität}

\section{Ausbildung}

In Punkt II, Absatz 2.1, der Anforderungen ist festgelegt: Der D-Arzt muss zum Führen der Deutschen Facharztbezeichnung Chirurgie oder Orthopäde und Unfallchirurg berechtigt sein und darüber hinaus über die Schwerpunktbezeichnung "spezielle Unfallchirurgie“ verfügen.

Wenn diese Anforderung strikt eingehalten wird, ist dies das Ende vieler chirurgischer Durchgangsarztpraxen, und die Zulassung kleinerer Krankenhäuser ist gefährdet oder deren Schließung ist bereits vollzogen. Hier stellt sich die Frage, ob dies beabsichtigt war.

Nachfolger mit der zusätzlichen Qualifikation neben der Facharztbezeichnung Chirurg oder Orthopäde und Unfallchirurg zu finden, welche gleichzeitig über die Bezeichnung "spezielle Unfallchirurgie“ verfügen, wird immer schwieriger werden. Wo sollen diese Kollegen noch zahlenmäßig ausreichend ausgebildet werden, wenn Altassistenten die Stellen wegen fehlender Perspektiven im niedergelassenen Bereich blockieren?

Auch die Patientenversorgung wird sehr darunter zu leiden haben, denn weite Wege und sehr lange Wartezeiten werden in den wenigen verbleibenden DArzt-Praxen die Regel sein.

Aus diesen Gründen wird an die Unfallversicherungsträger appelliert, die Anforderungen nicht $\mathrm{zu}$ stringent $\mathrm{zu}$ handhaben und bei Überprüfung derselben be- züglich der einzelnen Leistungserbringer zu differenzieren:

- Große unfallchirurgische Abteilungen könnten nur von einem Chirurgen mit der Zusatzbezeichnung ,spezielle Unfallchirurgie" geleitet werden.

- Bei Krankenhäusern der Grund- und Regelversorgung sollte der Nachweis der Facharztbezeichnung „Chirurg oder Orthopädie und Unfallchirurgie" genügen, Gleiches sollte für den Durchgangsarzt gelten.

Das Problem des Verletzungsartenverfahrens, der so genannten $\$ 6$-Fälle, welche eine hohe Struktur- und Prozessqualität erfordern, kann dadurch gelöst werden, dass diese in den großen unfallchirurgischen Kliniken versorgt werden bzw. das kleinere Krankenhaus oder der niedergelassene D-Arzt in der Praxis in Kooperation mit engmaschigen Kontrollen unter Einbeziehung der Berufshelfer die optimale Versorgung möglich machen. Vorraussetzung dafür ist jedoch, dass die Berufsgenossenschaften die Zahl der Berufshelfer erhöhen und diese häufiger in das Heilverfahren mit einbeziehen. Auch sollte die Kooperation der Arbeitgeber von den Berufsgenossenschaften vermehrt eingefordert werden.

\section{Sachliche Ausstattungen}

Ein weiterer Kritikpunkt ist die Anforderung IV, Abs. 3 mit den Unterpunkten 1-8. Dort werden sachliche Ausstattungen eingefordert, wie 2 Eingriffsräume für invasive Eingriffe, Sterilisationsräume, Röntgenraum mit einer Röntgenanlage min- 
Trauma Berufskrankh 2007 - 9 [Suppl 3]:

S333-S335

DOI 10.1007/s10039-007-1226-3

(c) Springer Medizin Verlag 2007

F. Kleinschmidt

Chirurgische

Organisationsformen

und berufsgenossenschaftliches Regelwerk. Was passt zusammen?

\section{Zusammenfassung}

Im vorliegenden Beitrag wird auf die Umsetzung des berufsgenossenschaftlichen Regelwerks aus der Sicht des niedergelassenen DArztes eingegangen. Dabei werden insbesondere die Schwierigkeiten bzw. Inkompatibilitäten mit modernen, chirurgischen Organisationsformen geschildert und Lösungsvorschläge gegeben. Abschließend wird auf Kommunikationsschwierigkeiten zwischen BG und niedergelassenen Ärzten eingegangen sowie das wichtige Problem der adäquaten Vergütung angesprochen.

\section{Schlüsselwörter}

Berufsgenossenschaftliches Regelwerk - DArzt - Chirurgische Organisation · Kommunikationsschwierigkeiten · Adäquate Vergütung

\section{Organisational forms in surgery and regulatory work of the employers' liability insurance associations. What goes with what?}

\begin{abstract}
This article deals with implementation of the employers'liability insurance association's regulatory work from the viewpoint of the doctor in private practice who is on emergency call-out. In particular, the difficulties or incompatibilities with modern organisational forms in surgery are described and some solutions are suggested. At the end there is a discussion of the difficulties in communication between employers' liability insurance association and doctors in private practice who are on call in other sectors also, such as the DALE UV system.
\end{abstract}

\section{Keywords}

Regulatory work of the employers' liability insurance association · Doctor on call . Organisation in surgery . Communication problems - Adequate remuneration destens der Anwendungsklasse II sowie ausreichende Einrichtungen zum Archivieren. Auch hier wäre eine Anpassung an moderne, zeitgemäße Organisationsformen wünschenswert:

- Das neue VÄG erlaubt es dem Arzt, Tätigkeiten an weiteren Orten außerhalb des ursprünglichen Vertragsarztsitzes zu verrichten (so genannte ausgelagerte Praxisräume). Es stellt sich daher die Frage, warum es die Berufsgenossenschaften beanstanden, wenn Kliniken und Praxen ihre Röntgenabteilung ausgelagert haben oder die Röntgenaufnahmen bei einem unmittelbar benachbarten Radiologen anfertigen lassen.

- Es ist ebenfalls nicht einzusehen, dass der niedergelassene Vertragsarzt 2 Eingriffsräume für invasive Eingriffe vorhalten soll, wenn er diese in einem ambulanten Operationszentrum angemietet hat.

- Sowohl für das Krankenhaus als auch den niedergelassenen Vertragsarzt sind die ausgelagerte Sterilisationseinheit und die elektronische Archivierung ohne große Archivräume heute durchaus gängige Praxis.

\section{Anforderung der persönlichen Versorgung}

Ein weiter Punkt, warum die chirurgischen Organisationsformen und das berufsgenossenschaftliche Regelwerk nicht mehr zusammenpassen, ist die Anforderung und die damit verbundene Verpflichtung, dass die durchgangsärztliche Tätigkeit persönlich und unter der Beachtung der Grundsätze der Sparsamkeit und Wirtschaftlichkeit ausgeübt wird. $\mathrm{Zu}$ diesen Punkt werden Herr Ritter aus Heidelberg sowie Herr Wirthl aus Mainz ausführlich referieren.

Nur der niedergelassene Durchgangsarzt kann diese Anforderungen erfüllen. In einer Klinik oder einem Krankenhaus ist dies unmöglich, denn wie soll der zum D-Arzt-Verfahren zugelassene Chefarzt neben seiner klinischen, stationären Tätigkeit ambulante Patienten persönlich versorgen können.

\section{Lösungsvorschläge}

Um die angesprochenen Probleme bei gleichzeitiger Aufrechterhaltung der Ver- sorgungsqualität zu lösen, sollte der Unfallversicherungsträger lediglich Anforderungen hinsichtlich der Struktur- und Prozessqualität stellen, wie

1. Der Verunfallte ist von einem D-Arzt zu versorgen oder ihm vorzustellen. Bei nächtlichen und feiertäglichen Unfällen ist der Patient für den nächsten Tag einzubestellen und einem DArzt zuzuweisen.

2. Die ausgelagerten Ambulanzen, Notfallambulanzen oder Notfallaufnahmen in einem Krankenhaus sollten unter eine Leitung gestellt werden. Mehrere D-Ärzte zu bestellen, ist unsinnig, dient lediglich einer personellen Versorgungssituation und ist bei einem guten Klinikleiter nicht erforderlich. Außerdem kann es zu erheblichen fachlichen Differenzen zwischen den zugelassenen D-Ärzten kommen, denn es ist allgemein bekannt: „1 Patient, 2 Ärzte und $3 \mathrm{Mei}$ nungen".

3. Während der ambulanten Weiterbehandlung sollte es dem zugelassenen D-Arzt gestattet sein, Leistungen zu delegieren, lediglich die Verantwortung muss bei ihm bleiben.

\section{Kommunikationsschwie- rigkeiten zwischen BG und niedergelassenen Ärzten}

Die Berufsgenossenschaften sind der Ansicht, dass ihre Anforderungen auch mit den heutigen chirurgischen Organisationsformen kompatibel sind, denn für sie gibt es in dieser Hinsicht keine Probleme. Diese sind aber vorhanden, genauso, wie es umgekehrt massive Schwierigkeiten beim DALE-UV-System gab und gibt: Der genannte Einführungstermin wurde nicht gehalten, die Organisation funktioniert an vielen Punkten z. T. heute noch nicht, so hapert es z. B. an der Weiterleitung der Berichte an die verschiedenen Berufsgenossenschaften oder die Hausärzte; personelle Unzulänglichkeiten aufgrund fehlender Weiterbildung in den Verwaltungen der einzelnen BG sowie die häufig zu bemerkende nachträgliche Anforderung von ausgedruckten D-Berichten seien beispielhaft genannt. 


\section{Vergütung}

In einem Schreiben an die Durchgangsärzte vom 24.01.07 kündigte der Landesverband Rheinland Westfalen eine $\mathrm{Ab}$ senkung der besonderen Kosten an und vollzog diese auch. Dies gilt speziell für die Krankenhäuser und hier die Spalte 3, aber auch bei den niedergelassenen Chirurgen wurde eine Senkung der Nebenkosten zum 01.03.07 vollzogen. Dabei wurde nicht berücksichtigt, dass seit 2000

- die Mehrwertsteuer erhöht wurde,

- die Energiekosten gestiegen sind,

- die regelmäßigen kostenpflichtigen Überwachungen (durch die Gesundheitsämter, durch den Regierungspräsidenten, durch den technischen Überwachungsverein usw.) zugenommen haben sowie

- die Inflationsrate angestiegen ist.

Nicht alle niedergelassenen Chirurgen haben die Möglichkeit, diese Durchlaufposten so preiswert zu halten, wie die Kollegen in Bayern. Auch sind z. T. nicht begründete und nicht nachvollziehbare Rechnungskürzungen erfolgt, hierbei seien insbesondere die Ziffer 6 und die Ziffer für konsiliarische Beratung erwähnt.

Absolut konträr sind das berufsgenossenschaftliche Regelwerk und die chirurgische Organisationsform in den Punkten, in welchen Verwaltungsmitarbeiter Sachentscheidungen nach internen Richtlinien bei der Rechnungskürzung und der weiteren Begleitung des Heilverfahrens treffen, die zum einen nicht dem Regelwerk entsprechen und zum anderen medizinisch unsinnig sind.

\section{Fazit}

Chirurgische Organisationsformen und berufsgenossenschaftliches Regelwerk sind auch oft kompatibel, aber in Zeiten verminderter Ressourcen und einer schwer zu verstehenden Gesundheitspolitik, die meiner Meinung nach zwingend in eine Staatsmedizin führt, müssen die Unfallversicherungsträger und die Leistungserbringer in Praxis und Klinik versuchen, Machbares, Wirtschaftliches und medizinisch Sinnvolles gemeinsam umzusetzen.

\section{Korrespondenzadresse}

\section{Dr. F. Kleinschmidt}

Eichendorffstraße 2,

50519 Möhnesee

Interessenkonflikt. Der korrespondierende Autor gibt an, dass kein Interessenkonflikt besteht. 